\title{
Model Identification for Robot Manipulators using Regressor-Free Adaptive Control
}

\author{
Rameez Hayat, Martin Buss \\ Chair of Automatic Control Engineering (LSR) \\ Technical University of Munich, Munich, Germany \\ Email: rameez.hayat@tum.de,mb@tum.de
}

\begin{abstract}
This paper proposes a regressor-free adaptive feedback-linearization control technique that does not require a model approximation or a regressor matrix. Adaptation in the proposed feedback process is acquired through an update law involving adjustment of less control parameters as compared to existing controllers. Under the given constraints, the closed-loop asymptotic stability of the proposed control law is verified using Lyapunov techniques. The proposed controller is compared with existing adaptive controllers on a two degree-of-freedom robot manipulator. Based on the new adaptive technique, the model parameters of the robotic arm are identified using adequate excitation trajectories. The proposed adaptive technique was validated through simulations and experiments.
\end{abstract}

\section{INTRODUCTION}

In a dynamic environment, robots are required to perform at high-speed. Mass production in industry is one such application. Manufacturing of product requires precise synchronization of robot's motion along different axis. To achieve high performance for a model-based control method, knowledge about manipulator dynamics is inevitable. Tracking a desired trajectory by the robot's end-effector at high-speed is an important challenge [1], [2].

Computed torque [3], [4] is the most extensively used method to design controllers for robotic arms. One advantage of this method is that it results in a feedback linearization of the non-linear system. However, disturbances, un-modeled dynamics and parameter uncertainties limit the applicability of computed torque methods. The dynamics of a robot depend on its inertia, friction and its geometry. The behavior of friction also plays a significant role in the tracking process [5], [6]. The parameters of a robot are roughly known from its design but direct measurement of the coefficients of physical parameters contain uncertainties due to the complexity of the manipulator. To overcome these problems, different controllers have been proposed such as robust control [7], [8], adaptive control [9][11], neural network-based force control [12], [13], model predictive control [14] and disturbance observer control [15].

Most of the adaptive controllers are regressor-based methods [4], [16], which approximate the system dynamics using a regressor matrix that is linear in the unknown parameters. This technique, however, requires estimation of the inertia matrix and output acceleration. To overcome these problems Slotine and $\mathrm{Li}$ [16] proposed a regressor based adaptive control law that does not rely on these two requirements. Finding the regressor matrix, especially for high degree-of-freedom
(DOF) robotic arms, is a sophisticated and complex task. The function approximation technique (FAT) based adaptive controller [9]-[11] replaces the regressor matrix with a linear approximated function that can be represented in terms of orthogonal basis functions. By increasing the order of the basis, the approximation of the system parameters improves. To achieve high performance in terms of tracking errors using computed torque methods, the regressor matrix or FAT-based approximation of system dynamics should perfectly match the original model. However, all of the above adaptive control methods use Lyapunov theory to find the control parameters. For the existing adaptive controllers, it will be shown in later sections that this Lyapunov equation only guarantees stability but it does not assure approximation of the system dynamics.

The contribution of this article is twofold. First, the paper derives a new adaptive control method that assures the convergence of the estimated model towards the original system dynamics using few tuning parameters. Using computed torque, this yields a perfect feedback linearization due to the accurate estimation of system coefficients. Once the mismatch between original and approximated model is resolved, a PD controller can be implemented to achieve high-performance. The second contribution of the paper is to identify system parameters using the proposed adaptive control. The dynamical model of a robot manipulator is usually formulated using the Euler-Lagrange formulation. Thus a mathematical model of the system is known and the uncertainties lie only in the constant coefficients. A lot of research effort focused on the identification of these system parameters [17], [18]. As it will be shown in the stability proof, the estimated system dynamics approaches the real dynamics. Using this fact, the unknown manipulator parameters can be approximated using leastsquare-approximation (LS). A 2-DOF robotic manipulator is used to demonstrate the proposed technique.

\section{CONTROL TECHNIQUES}

\section{A. Feedback Linearization}

The dynamics of an $n$-link robotic manipulator can be represented using Euler-Lagrange equations as [4]:

$$
\boldsymbol{M}(\boldsymbol{q}) \ddot{\boldsymbol{q}}+\boldsymbol{C}(\boldsymbol{q}, \dot{\boldsymbol{q}}) \dot{\boldsymbol{q}}+\boldsymbol{G}(\boldsymbol{q})+\boldsymbol{F} \dot{\boldsymbol{q}}=\boldsymbol{\tau},
$$

where $\boldsymbol{M}(\boldsymbol{q}) \in \mathbb{R}^{n \times n}$ is a symmetric positive definite inertia matrix, $\boldsymbol{C}(\boldsymbol{q}, \dot{\boldsymbol{q}}) \dot{\boldsymbol{q}} \in \mathbb{R}^{n \times 1}$ is a vector of centrifugal and Coriolis terms, $\boldsymbol{G} \in \mathbb{R}^{n \times 1}$ contains the gravitational terms acting 
on the robot, $\boldsymbol{F} \in \mathbb{R}^{n \times n}$ is a diagonal matrix representing viscous friction, $\boldsymbol{q} \in \mathbb{R}^{n \times 1}$ is a vector representing the joint angles and $\tau \in \mathbb{R}^{n \times 1}$ is a vector of input torques applied on each joint.

If the matrices mentioned above are accurately known, a PD controller with feedback linearization can be implemented to get a simple second-order linear differential equation. By adjusting the velocity and proportional gains of the PD controller, the robot can be manipulated according to the desired response. Let the acceleration error be:

$$
\ddot{e}=\ddot{q}-\ddot{q}_{d}
$$

where $\ddot{\boldsymbol{q}}_{d}$ is the desired acceleration. Inserting (2) in (1) yields:

$$
\boldsymbol{\tau}=\boldsymbol{M}\left(\ddot{\boldsymbol{q}}_{d}+\ddot{\boldsymbol{e}}\right)+\boldsymbol{N}+\boldsymbol{F} \dot{\boldsymbol{q}},
$$

where $\boldsymbol{N}=\boldsymbol{C}(\boldsymbol{q}, \dot{\boldsymbol{q}}) \dot{\boldsymbol{q}}+\boldsymbol{G}(\boldsymbol{q})$. The above equation allows feedback linearization of the overall system. If the system dynamics are known and let $\ddot{\boldsymbol{e}}=-\boldsymbol{K}_{v} \dot{\boldsymbol{e}}-\boldsymbol{K}_{p} \boldsymbol{e}$, (1) becomes:

$$
\ddot{e}+\boldsymbol{K}_{v} \dot{e}+\boldsymbol{K}_{p} \boldsymbol{e}=\mathbf{0} .
$$

For a non oscillatory response, the PD parameters $\boldsymbol{K}_{v} \in \mathbb{R}^{n \times n}$ and $\boldsymbol{K}_{p} \in \mathbb{R}^{n \times n}$, are set to yield an over-damped or critically damped system. Taking $\ddot{e}$ from (4), the required torque is:

$$
\boldsymbol{\tau}=\boldsymbol{M}\left(\ddot{\boldsymbol{q}}_{d}-\boldsymbol{K}_{v} \dot{\boldsymbol{e}}-\boldsymbol{K}_{p} \boldsymbol{e}\right)+\boldsymbol{N}+\boldsymbol{F} \dot{\boldsymbol{q}},
$$

In a current controlled robotic manipulator, the equation for feedback linearization is:

$$
\boldsymbol{n}_{r} \boldsymbol{k}_{t} \boldsymbol{i}_{a}=\boldsymbol{M}\left(\ddot{\boldsymbol{q}}_{d}-\boldsymbol{K}_{v} \dot{\boldsymbol{e}}-\boldsymbol{K}_{p} \boldsymbol{e}\right)+\boldsymbol{N}+\boldsymbol{F} \dot{\boldsymbol{q}},
$$

where $\boldsymbol{k}_{t}$ is the torque constant, $\boldsymbol{i}_{a}$ is the armature current and $\boldsymbol{n}_{r}$ represents the gear ratio.

\section{B. Lyapunov based Adaptive Control}

For the ideal scenario, where the system dynamics are exactly known, the dynamical equations of a robot manipulator reduce to (4), and a simple PD controller can be used to achieve high performance. However, as discussed in section I, available system parameters in general are not precise, so:

$$
\boldsymbol{\tau}=\hat{\boldsymbol{M}}\left(\ddot{\boldsymbol{q}}_{d}-\boldsymbol{K}_{v} \dot{\boldsymbol{e}}-\boldsymbol{K}_{p} \boldsymbol{e}\right)+\hat{\boldsymbol{N}}+\hat{\boldsymbol{F}} \dot{\boldsymbol{q}},
$$

where $\hat{\boldsymbol{M}}, \hat{\boldsymbol{N}}$ and $\hat{\boldsymbol{F}}$ are estimates for $\boldsymbol{M}, \boldsymbol{N}$ and $\boldsymbol{F}$, respectively. Using the estimated torque (6), the error dynamics in (4) is:

$$
\ddot{\boldsymbol{e}}+\boldsymbol{K}_{v} \dot{\boldsymbol{e}}+\boldsymbol{K}_{p} \boldsymbol{e}=-\hat{\boldsymbol{M}}^{-\mathbf{1}}(\tilde{\boldsymbol{M}} \ddot{\boldsymbol{q}}+\tilde{\boldsymbol{N}}+\tilde{\boldsymbol{F}} \dot{\boldsymbol{q}}),
$$

where $\tilde{M}=\hat{M}-\boldsymbol{M}, \tilde{\boldsymbol{N}}=\hat{\boldsymbol{N}}-\boldsymbol{N}$ and $\tilde{\boldsymbol{F}}=\hat{\boldsymbol{F}}-\boldsymbol{F}$. The structure of a robot manipulator is precisely known from Euler-Lagrange formulation and the uncertainties appear only in the constant coefficients of the dynamical model.

Following the regressor-based adaptive approach [19], the system dynamics are described by:

$$
\boldsymbol{Y}(\boldsymbol{q}, \dot{\boldsymbol{q}}, \ddot{\boldsymbol{q}}) \boldsymbol{p}=\boldsymbol{M}(\boldsymbol{q}) \ddot{\boldsymbol{q}}+\boldsymbol{N}(\boldsymbol{q}, \dot{\boldsymbol{q}})+\boldsymbol{F} \dot{\boldsymbol{q}}
$$

where $\boldsymbol{Y}(\boldsymbol{q}, \dot{\boldsymbol{q}}, \ddot{\boldsymbol{q}})$ is a non-linear, known regression matrix with proper dimension and $\boldsymbol{p}$ is the unknown, constant parameter vector. Using the regressor-based approach, (7) becomes:

$$
\ddot{\boldsymbol{e}}+\boldsymbol{K}_{v} \dot{\boldsymbol{e}}+\boldsymbol{K}_{p} \boldsymbol{e}=-\hat{\boldsymbol{M}}^{-1} \boldsymbol{Y}(\boldsymbol{q}, \dot{\boldsymbol{q}}, \ddot{\boldsymbol{q}}) \tilde{\boldsymbol{p}},
$$

where $\tilde{\boldsymbol{p}}=\hat{\boldsymbol{p}}-\boldsymbol{p}$ and $\hat{\boldsymbol{p}}$ is the estimated value of $\boldsymbol{p}$. In order to track the desired behaviour in (4), we need an update law, which drives $\hat{\boldsymbol{p}}$ towards $\boldsymbol{p}$. Let $\boldsymbol{x}=\left[\begin{array}{ll}\boldsymbol{e}^{T} & \dot{\boldsymbol{e}}^{T}\end{array}\right]^{\boldsymbol{T}}$ be the state error vector, then (9) can be written as:

$$
\dot{\boldsymbol{x}}=\boldsymbol{A} \boldsymbol{x}-\boldsymbol{B} \boldsymbol{M}^{-1} \boldsymbol{Y}(\boldsymbol{q}, \dot{\boldsymbol{q}}, \ddot{\boldsymbol{q}}) \tilde{\boldsymbol{p}}
$$

where

$$
\boldsymbol{A}=\left[\begin{array}{cc}
\mathbf{0} & \boldsymbol{I}_{n} \\
-\boldsymbol{K}_{p} & -\boldsymbol{K}_{v}
\end{array}\right] \in \mathbb{R}^{2 n \times 2 n}, \boldsymbol{B}=\left[\begin{array}{c}
\mathbf{0} \\
\boldsymbol{I}_{n}
\end{array}\right] \in \mathbb{R}^{2 n \times n}
$$

and $\boldsymbol{I}_{n}$ is the identity matrix. Consider the following Lyapunov function:

$$
V(\boldsymbol{x}, \tilde{\boldsymbol{p}})=\frac{1}{2} \boldsymbol{x}^{T} \boldsymbol{P}_{1} \boldsymbol{x}+\frac{1}{2} \tilde{\boldsymbol{p}}^{T} \boldsymbol{P}_{2} \tilde{\boldsymbol{p}},
$$

where $\boldsymbol{P}_{1}$ and $\boldsymbol{P}_{2}$ are symmetric, positive definite matrices. Taking the derivative of (11) along (10) yields:

$$
\dot{V}=-\frac{1}{2} \boldsymbol{x}^{T} \boldsymbol{Q} \boldsymbol{x}-\tilde{\boldsymbol{p}}^{T}\left[\left(\hat{\boldsymbol{M}}^{-1} \boldsymbol{Y}\right)^{T} \boldsymbol{B}^{T} \boldsymbol{P}_{1} \boldsymbol{x}+\boldsymbol{P}_{2} \dot{\hat{\boldsymbol{p}}}\right] .
$$

Since $\boldsymbol{A}$ is a Hurwitz matrix [14], there exists a positive definite matrix $\boldsymbol{Q}=\boldsymbol{Q}^{T}$ satisfying $\boldsymbol{A}^{T} \boldsymbol{P}_{1}+\boldsymbol{P}_{1} \boldsymbol{A}=-\boldsymbol{Q}$. In order to cancel some terms, the update law for $\hat{\boldsymbol{p}}$ is selected as:

$$
\dot{\hat{\boldsymbol{p}}}=-\boldsymbol{P}_{2}^{-1}\left(\hat{\boldsymbol{M}}^{-1} \boldsymbol{Y}\right)^{T} \boldsymbol{B}^{T} \boldsymbol{P}_{1} \boldsymbol{x} .
$$

Substituting $\dot{\hat{\boldsymbol{p}}}$ in (12), the Lyapunov function derivative becomes $\dot{V}=-\frac{1}{2} \boldsymbol{x}^{T} \boldsymbol{Q} \boldsymbol{x} \leqslant 0$, which ensures asymptotic tracking of the reference trajectory.

Inverse of the inertia matrix often may not be possible because of singularity issue. Also the joint acceleration required in the regressor-based method contains noise. To avoid the use of $\hat{M}^{-1}$ and $\ddot{\boldsymbol{q}}$, Slotine et al. [16] reconfigured the system dynamics by considering $s=\dot{e}+\triangle e$, where $\triangle \in \mathbb{R}^{n \times n}$ is a diagonal matrix with positive eigenvalues. Using this new formulation, (1) takes the following form:

$$
\boldsymbol{M} \dot{s}+\boldsymbol{N}+\boldsymbol{F} \boldsymbol{s}+\boldsymbol{M} \dot{\boldsymbol{v}}+\boldsymbol{F} \boldsymbol{v}=\boldsymbol{\tau},
$$

$\boldsymbol{v}=\dot{\boldsymbol{q}}_{d}-\triangle \boldsymbol{e}$. Taking the torque $\boldsymbol{\tau}$ as $\boldsymbol{\tau}=\hat{\boldsymbol{M}} \dot{\boldsymbol{v}}+\hat{\boldsymbol{F}} \boldsymbol{v}+\hat{\boldsymbol{N}}-$ $\boldsymbol{K}_{D} \boldsymbol{s}=\boldsymbol{Y}(\boldsymbol{q}, \dot{\boldsymbol{q}}, \boldsymbol{v}, \dot{\boldsymbol{v}}) \hat{\boldsymbol{p}}$, , where $\boldsymbol{K}_{D}$ is a positive definite matrix, (14) takes the form:

$$
\boldsymbol{M} \dot{\boldsymbol{s}}+\boldsymbol{F} \boldsymbol{s}+\boldsymbol{K}_{D} \boldsymbol{s}=-\boldsymbol{Y}(\boldsymbol{q}, \dot{\boldsymbol{q}}, \boldsymbol{v}, \dot{\boldsymbol{v}}) \tilde{\boldsymbol{p}} .
$$

Following the same steps as before, we obtain the following formulation for the update law of $\hat{\boldsymbol{p}}$ :

$$
\dot{\hat{\boldsymbol{p}}}=-\boldsymbol{P}_{2}^{-1} \boldsymbol{Y}^{T}(\boldsymbol{q}, \dot{\boldsymbol{q}}, \boldsymbol{v}, \dot{\boldsymbol{v}}) \boldsymbol{s} .
$$

Huang et al. [11] proposed a FAT-based adaptive controller that does not require the regressor matrix to acquire the control parameters. The matrices $\boldsymbol{M}, \boldsymbol{N}$ and $\boldsymbol{F}$ are approximated by weighted sums of some orthogonal linear functions:

$$
\boldsymbol{M}=\boldsymbol{W}_{M}^{T} \boldsymbol{Z}_{M}, \quad \boldsymbol{F}=\boldsymbol{W}_{F}^{T} \boldsymbol{Z}_{F}, \quad \boldsymbol{N}=\boldsymbol{W}_{N}^{T} \boldsymbol{Z}_{N},
$$


where $\boldsymbol{W}_{(.)}$is a weighting matrix and $\boldsymbol{Z}_{(.)}$is a matrix of basis functions. In the above equation, approximation errors are ignored. Based on the Lyapunov function:

$$
\begin{aligned}
V=\frac{1}{2} \boldsymbol{s}^{T} \boldsymbol{M} \boldsymbol{s}+ & \frac{1}{2} \operatorname{tr}\left(\tilde{\boldsymbol{W}}_{M}^{T} \boldsymbol{Q}_{M} \tilde{\boldsymbol{W}}_{M}+\tilde{\boldsymbol{W}}_{F}^{T} \boldsymbol{Q}_{F} \tilde{\boldsymbol{W}}_{F}\right. \\
& \left.+\tilde{\boldsymbol{W}}_{N}^{T} \boldsymbol{Q}_{N} \tilde{\boldsymbol{W}}_{N}\right),
\end{aligned}
$$

the update laws are derived as [11]:

$$
\begin{aligned}
& \dot{\hat{\boldsymbol{W}}}_{M}=-\boldsymbol{Q}_{M}^{-1} \boldsymbol{Z}_{M} \dot{\boldsymbol{v}} \boldsymbol{s}^{T} \\
& \dot{\hat{\boldsymbol{W}}}_{F}=-\boldsymbol{Q}_{F}^{-1} \boldsymbol{Z}_{F} \boldsymbol{v} \boldsymbol{s}^{T} \\
& \dot{\hat{\boldsymbol{W}}}_{N}=-\boldsymbol{Q}_{N}^{-1} \boldsymbol{Z}_{N} \boldsymbol{s}^{T} .
\end{aligned}
$$

To further improve the control law, Kai et al. [9] replaced $\boldsymbol{M}^{-1} \boldsymbol{Y}(\boldsymbol{q}, \dot{\boldsymbol{q}}, \ddot{\boldsymbol{q}}) \boldsymbol{p}$ by $\boldsymbol{\psi}$, where $\boldsymbol{\psi}=\boldsymbol{W}^{T} \boldsymbol{Z}$. Following the same procedure for finding the control law by analyzing the Lyapunov function, we obtain":

$$
\dot{\hat{\boldsymbol{W}}}=-\boldsymbol{Q}^{-1} \boldsymbol{Z} \boldsymbol{x}^{T} \boldsymbol{P} \boldsymbol{B},
$$

where $\boldsymbol{P} \in \mathbb{R}^{2 n \times 2 n}$ is a positive definite matrix. The FATbased approach requires large computational cost because of large number of a orthogonal basis functions.

\section{PROPOSED ADAPTIVE TECHNIQUE}

In all of the above techniques, Lyapunov theory is used to derive the update laws. However these adaptive techniques only confirm the error to approach zero but they do not guarantee how fast $\hat{\boldsymbol{p}}$ would approach $\boldsymbol{p}$, if it converges at all. As can be seen from $\dot{V}=-1 / 2 \boldsymbol{x}^{T} \boldsymbol{Q} \boldsymbol{x}$, only $\boldsymbol{x}$ is decreasing with time but there is no information about $\tilde{p}$ decreasing with time. To force $\hat{\boldsymbol{p}} \rightarrow \boldsymbol{p}$, we propose a simple adaptive approach that require less tuning parameters compared to Lyapunovbased adaptive techniques. Moreover, the proposed technique also guarantee the convergence of modeling errors to zero, which is then used to identify the system parameters.

Let $\phi=\boldsymbol{M}(\boldsymbol{q}) \ddot{\boldsymbol{q}}+\boldsymbol{N}(\boldsymbol{q}, \dot{\boldsymbol{q}})+\boldsymbol{F} \dot{\boldsymbol{q}}-\ddot{\boldsymbol{q}}+\tau_{\boldsymbol{a}}$, where $\tau_{\boldsymbol{a}}$ represents disturbances and unmodeled parameters. Then the input torque is selected as [9]:

$$
\boldsymbol{\tau}=\boldsymbol{\phi}+\ddot{\boldsymbol{q}}_{d}-\boldsymbol{K}_{v} \dot{\boldsymbol{e}}-\boldsymbol{K}_{p} \boldsymbol{e}
$$

Using (19), equation (4) becomes:

$$
\ddot{e}+\boldsymbol{K}_{v} \dot{\boldsymbol{e}}+\boldsymbol{K}_{p} \boldsymbol{e}=\hat{\boldsymbol{\phi}}-\boldsymbol{\phi},
$$

where $\hat{\phi}$ is the estimate of $\phi$. To make $\hat{\phi}$ converge to $\phi$ and thus $e \rightarrow 0$, we introduce the following differential equation:

$$
\sum_{i=0}^{m} a_{i} \frac{d^{i} \tilde{\boldsymbol{\phi}}}{d t^{i}}=\boldsymbol{f}(\boldsymbol{e}, \dot{\boldsymbol{e}}),
$$

where the system mismatch $\tilde{\boldsymbol{\phi}}$ is $\hat{\boldsymbol{\phi}}-\boldsymbol{\phi}, a_{m}=1$ and $\boldsymbol{f}(\boldsymbol{e}, \dot{\boldsymbol{e}})$ is a function of the errors that will be evaluated in the stability subsection. If we consider $\boldsymbol{f}(\boldsymbol{e}, \dot{\boldsymbol{e}})$ to be zero, then depending on the values of $a_{i}$ in equation (21), $\tilde{\phi}$ will eventually tends towards zero. The only purpose of $\boldsymbol{f}(\boldsymbol{e}, \dot{\boldsymbol{e}})$ is to keep the system stable as proved in the next subsection. To avoid the

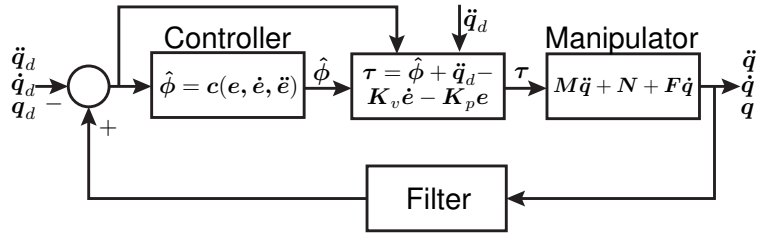

Fig. 1: Adaptive Feedback linearization of robot manipulator. $\boldsymbol{c}(\boldsymbol{e}, \dot{e}, \ddot{e})$ can be derived from the control law given in (22).

overall effect of poles of equation (21), the coefficients $a_{i}$ are selected to get a small time constant compared to the PD controller (19). In general, the poles of (21) should be at least five times faster than the closed-loop poles in (4) to make sure that $\hat{\phi}$ approaches $\phi$ without effecting the performance of the PD controller. Fig. 1 shows the block diagram of the proposed adaptive control technique.

To evaluate the value of $\hat{\phi}$ at every time instance, which is then used to determine the torque vector in (19), $\phi$ is approximated by a polynomial of order $m-1$ using Taylor series. Using (20) and (21), the update law becomes:

$$
\frac{d^{m} \hat{\boldsymbol{\phi}}}{d t^{m}}=-\sum_{i=0}^{m-1} a_{i} \frac{d^{i} \boldsymbol{X}}{d t^{i}}+\boldsymbol{f}(\boldsymbol{e}, \dot{\boldsymbol{e}}),
$$

where $\boldsymbol{X}=\ddot{\boldsymbol{e}}+\boldsymbol{K}_{v} \dot{\boldsymbol{e}}+\boldsymbol{K}_{p} \boldsymbol{e}$. Note that the update law does not require a regression matrix or FAT evaluation. It can very easily be implemented with low computational cost. To make a better Taylor approximation of $\phi$, it is desired to select a high value of $m$.

\section{A. Stability}

In this section, not only the asymptotic stability of the system is proved but also the convergence of $\hat{\phi}$ to $\phi$ using (22).

Theorem 1 (Stability): Let $\boldsymbol{K} \boldsymbol{x}=\boldsymbol{f}(\boldsymbol{e}, \dot{\boldsymbol{e}})$, then the proposed adaptive controller satisfy the asymptotic stability of the system if $\boldsymbol{K}=-\boldsymbol{P}_{2}{ }^{-1} \boldsymbol{B}^{T} \boldsymbol{P}_{1}$.

Proof: To prove the stability of the proposed technique, Lyapunov theory is used. Note that the techniques discussed in section II-B used Lyapunov theory to design the adaptive controller. While in the proposed technique, Lyapunov is only used to proof the stability of the adaptive technique of section III. The differential equation (21) can be written in matrix form as:

$$
\dot{z}=\boldsymbol{H} \boldsymbol{z}+\boldsymbol{K} \boldsymbol{x},
$$

where $\boldsymbol{z}=\left[\begin{array}{llll}\tilde{\boldsymbol{\phi}} & \dot{\tilde{\phi}} & \ldots & \dot{\tilde{\phi}}^{m-1}\end{array}\right]^{T} \in \mathbb{R}^{m n \times 1}$ and $\boldsymbol{H} \in$ $\mathbb{R}^{m n \times m n}$ is the Jordan canonical form of the left hand side of (21). Similarly (20) can be expressed in the state space form as:

$$
\dot{\boldsymbol{x}}=\boldsymbol{A} \boldsymbol{x}+\boldsymbol{B} \boldsymbol{z}
$$

where $\boldsymbol{A}$ and $\boldsymbol{x}$ are the same as in (10) and:

$$
\boldsymbol{B}=\left[\begin{array}{cccc}
0 & 0 & \ldots & 0 \\
\boldsymbol{I}_{\boldsymbol{n}} & 0 & \ldots & 0
\end{array}\right] \in \mathbb{R}^{2 n \times m n}
$$




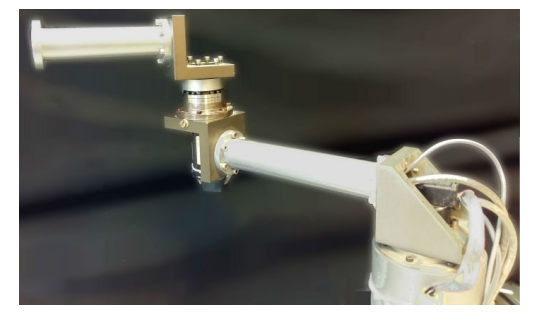

Fig. 2: Two-DOF serial robot used in experiments.

Let $V(\boldsymbol{x}, \boldsymbol{z})=\frac{1}{2} \boldsymbol{x}^{T} \boldsymbol{P}_{1} \boldsymbol{x}+\frac{1}{2} \boldsymbol{z}^{T} \boldsymbol{P}_{2} \boldsymbol{z}$, where $\boldsymbol{P}_{1}$ and $\boldsymbol{P}_{2}$ are symmetric, positive definite matrices. The derivative of this Lyapunov function is:

$$
\dot{V}=-\boldsymbol{x}^{T} \boldsymbol{Q}_{1} \boldsymbol{x}-\boldsymbol{z}^{T} \boldsymbol{Q}_{2} \boldsymbol{z}+2 \boldsymbol{z}^{T}\left(\boldsymbol{B}^{T} \boldsymbol{P}_{1}+\boldsymbol{P}_{2} \boldsymbol{K}\right) \boldsymbol{x} .
$$

As $\boldsymbol{A}$ and $\boldsymbol{H}$ are stable matrices, $\boldsymbol{A}^{T} \boldsymbol{P}_{1}+\boldsymbol{P}_{1} \boldsymbol{A}=-\boldsymbol{Q}_{1}$ and $\boldsymbol{H}^{T} \boldsymbol{P}_{2}+\boldsymbol{P}_{2} \boldsymbol{H}=-\boldsymbol{Q}_{2}$ with positive definite matrices $\boldsymbol{Q}_{1}$ and $\boldsymbol{Q}_{2}$. The matrix $\boldsymbol{K}$ with appropriate dimensions can easily be evaluated from the above equation as:

$$
\boldsymbol{K}=-\boldsymbol{P}_{2}^{-1} \boldsymbol{B}^{T} \boldsymbol{P}_{1} \text {. }
$$

Model identification is only possible if $\boldsymbol{z} \rightarrow \mathbf{0}$ as explained in the next section. From the stability analysis above, it is proved that both $\boldsymbol{x}$ and $\boldsymbol{z}$ approach zero because $\dot{V}=-\boldsymbol{x}^{T} \boldsymbol{Q}_{1} \boldsymbol{x}-$ $\boldsymbol{z}^{T} \boldsymbol{Q}_{2} \boldsymbol{z} \leqslant 0$. The speed of convergence of $\tilde{\boldsymbol{\phi}}$ depends on the values of $a_{i}$. But higher gain of (21) require higher values of torques, which in practice cannot be achieved if the torque is higher than the power limits of the motors. So, $a_{i}$ is selected such that the mismatch $\tilde{\phi}$ tends to zero much faster than $e$ without violating the power limits of the motors.

\section{MODEL IDENTIFICATION}

The structure of robot manipulators can be evaluated using the Euler-Lagrange equation and the uncertainties lie only in the constant coefficients of the system parameters. Using the proposed controller, $\boldsymbol{z} \rightarrow \mathbf{0}$, we eventually get:

$$
\ddot{e}+\boldsymbol{K}_{v} \dot{e}+\boldsymbol{K}_{p} \boldsymbol{e}=\mathbf{0} .
$$

To find the unknown parameters, take the values of $\hat{\phi}$ whenever the above equation is true at each time step (since $\phi=\hat{\phi}$ at that sample). Once $\phi$ is known, least square technique can be used to determine the system parameters. The essential requirement for identifying the system model using LS is having a proper excitation trajectory. To identify model parameters experimentally, it is necessary to have position, velocity and acceleration at each joint to get a rich regressor matrix. Note that this regressor matrix is not related to the adaptive control. The excitation trajectory can be chosen as a finite sum of harmonic functions to avoid noise [18]:

$$
q_{i}(t)=\sum_{l=1}^{N}\left[a_{l}^{i} \sin \left(\omega_{f} l t\right)+b_{l}^{i} \cos \left(\omega_{f} l t\right)\right],
$$

where $a_{l}^{i}$ and $b_{l}^{i}$ are the magnitudes of $l^{\text {th }}$ harmonic of the $i^{\text {th }}$ joint angle. Once a large number of data points for $\hat{\phi}$

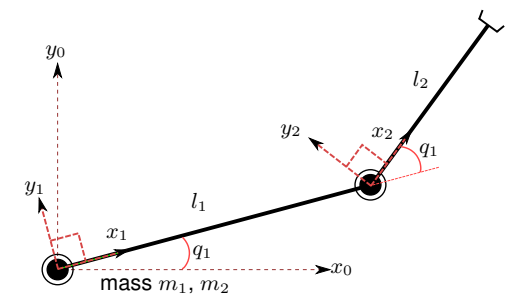

Fig. 3: Coordinate frames of a 2-DOF planar robot.

is available, which in our experiments are nearly 5000 data points, LS can be applied to estimate the model parameters [20]:

$$
\begin{gathered}
\mathcal{Y} \hat{\boldsymbol{p}}=\overline{\boldsymbol{B}} \\
\hat{\boldsymbol{p}}=\left(\mathcal{Y}^{T} \mathcal{Y}\right)^{-1} \mathcal{Y}^{T} \overline{\boldsymbol{B}},
\end{gathered}
$$

where, $\mathcal{Y}=\left[\begin{array}{llll}\boldsymbol{Y}_{1}^{T} & \boldsymbol{Y}_{2}^{T} & \ldots & \boldsymbol{Y}_{N}^{T}\end{array}\right]^{T}, \overline{\boldsymbol{B}}=\left[\begin{array}{llll}\hat{\boldsymbol{\phi}}_{1}^{T} & \hat{\boldsymbol{\phi}}_{2}^{T} & \ldots & \hat{\boldsymbol{\phi}}_{N}^{T}\end{array}\right]^{T}$, and $\boldsymbol{N}$ is the total number of sampled data points. The columns of the matrix $\mathcal{Y}$ should be linearly independent for LS to accurately approximate the parameters. The estimation process can still be improved using total least square approximation, which also considers uncertainties in the regressor matrix.

\section{RESULTS AND ANALYSIS}

The proposed adaptive technique and all of the previous techniques mentioned in section II-B are implemented on a 2-DOF robot manipulator as shown in Fig. 2 and 3. A circular desired trajectory with a radius of $0.15 \mathrm{~m}$ and a center at $0.24 \widehat{x}+0.24 \widehat{y} \mathrm{~m}$ is considered, where $\widehat{x}$ and $\widehat{y}$ are unit vectors in the $x$ and $y$ directions respectively. The matrices $\boldsymbol{M}(\boldsymbol{q}), \boldsymbol{N}(\boldsymbol{q}, \dot{\boldsymbol{q}})$ and $\boldsymbol{F}$ for the 2-DOF manipulator are:

$$
\begin{gathered}
\boldsymbol{M}=\left[\begin{array}{cc}
m_{11}+m_{11}^{\prime} \cos q_{2} & m_{12}+m_{12}^{\prime} \cos q_{2} \\
m_{21}+m_{21}^{\prime} \cos q_{2} & m_{22}
\end{array}\right], \\
\boldsymbol{N}=\left[\begin{array}{c}
-n_{11} \sin q_{2} \dot{q}_{2}^{2}-n_{12} \sin q_{2} \dot{q}_{1} \dot{q}_{2} \\
n_{21} \sin q_{2} \dot{q}_{1}^{2}
\end{array}\right], \boldsymbol{F}=\left[\begin{array}{cc}
f_{11} & 0 \\
0 & f_{22}
\end{array}\right]
\end{gathered}
$$

, where the unknown constant coefficients in the above matrices depend on the physical parameters of the robot manipulator as shown in Table. I. The planar robot lies in the horizontal plan, thus gravity terms are zero.

\section{A. Simulation results}

Simulation results are shown in Fig. 4-8. For a regressorbased method shown in Fig. 4, inverse of the inertial matrix limits the overall performance of the controller. The matrices in (13) are chosen as $\boldsymbol{P}_{2}=\boldsymbol{I}$ and:

$$
\boldsymbol{P}_{1}=\left[\begin{array}{cccc}
6.05 & 0 & 0.008 & 0 \\
0 & 2.13 & 0 & 0.0025 \\
0.008 & 0 & 0.01 & 0 \\
0 & 0.0025 & 0 & 0.01
\end{array}\right]
$$

Fig. 4-a and 4-c represent $\boldsymbol{Y}(\boldsymbol{q}, \dot{\boldsymbol{q}}, \ddot{\boldsymbol{q}}) \boldsymbol{p}$ (real) and $\boldsymbol{Y}(\boldsymbol{q}, \dot{\boldsymbol{q}}, \ddot{\boldsymbol{q}}) \hat{\boldsymbol{p}}$ (estimated) taken from (8) and (9) while their difference is shown in Fig. 8. An upper bound on the joint torques should 
be applied to avoid singularity problems. Although Fig. 4-b and 4-d show that the joint angles and its derivatives tries to follow the desired trajectory to satisfy the Lyapunov criteria, there is no guarantee on $\boldsymbol{Y}(\boldsymbol{q}, \dot{\boldsymbol{q}}, \ddot{\boldsymbol{q}}) \tilde{\boldsymbol{p}}$ to approach zero (see Fig. 8).

The approach by Slotine et al. in Fig. 5 shows improved performance as compared to the simple regression because the inverse of the inertial matrix is not required. However there are various design parameters in (14) and (16), which require trial and error to adjust the controller. From (16), $\boldsymbol{P}_{2}=\boldsymbol{I}$ and:

$$
\triangle=\left[\begin{array}{cc}
10 & 0 \\
0 & 10
\end{array}\right], \quad \boldsymbol{K}_{D}=\left[\begin{array}{cc}
200 & 0 \\
0 & 150
\end{array}\right]
$$

The FAT-based controller in Fig. 6 has the advantage of using linear approximated models of the system. However, this technique uses large matrices for the approximation thus increasing the complexity of the controller. Also some information is lost because of linearization. The design parameters of the FATbased approach are taken from [9].

As shown in Fig. 8, $\boldsymbol{Y}(\boldsymbol{q}, \dot{\boldsymbol{q}}, \ddot{\boldsymbol{q}}) \tilde{\boldsymbol{p}}, \boldsymbol{Y}(\boldsymbol{q}, \dot{\boldsymbol{q}}, \boldsymbol{v}, \dot{\boldsymbol{v}}) \tilde{\boldsymbol{p}}$ and $\boldsymbol{W}_{(.)}^{T} \boldsymbol{Z}_{(.)}$for regressor-based, Slotine and FAT-based approaches respectively never converge to zero. For this reason, these approaches cannot be used to identify the system coefficients. In the proposed adaptive method (Fig. 7), if one can ignore values at higher derivatives of the original model $\phi$, the error $\tilde{\phi}$ converges to zero as proved in section III-A and also evident from Fig. 8. Considering a critically damped system, $a_{i}$ and $\boldsymbol{f}(\boldsymbol{e}, \dot{\boldsymbol{e}})$ can be easily evaluated using (22) and (23). $\boldsymbol{P}_{1}$ and $\boldsymbol{P}_{2}$ are taken as identity matrices.

\section{B. Experimental results}

The experimental results for all of the controllers are shown in Fig. 9. As explained in section III, to avoid noise, equation (21) is taken as a first order differential equation, which is $m=1$. A low-pass filter is also used to remove noise in the joint acceleration. Apart from the PD gains, the proposed technique requires only one variable $a$ to adjust the adaptive controller. However, a large value of $a$ gives higher torques, which introduces high frequency oscillation. In experiments $a=80$, thus ensuring that the performance of PD controller is not disturbed by the pole of equation (21). The values for the PD gain are:

$$
\boldsymbol{K}_{v}=\left[\begin{array}{cc}
10 & 0 \\
0 & 10
\end{array}\right], \quad \boldsymbol{K}_{p}=\left[\begin{array}{cc}
100 & 0 \\
0 & 100
\end{array}\right]
$$

The poles for the PD gain are at -10 , while $a$ is selected eight times faster than the poles of PD controller. Because of the reason that the mismatch tends to zero in the proposed adaptive controller, the end-effector follows the trajectory satisfying equation (4) as clear from Fig. 9. Note that the proposed adaptive control technique also deals with disturbances because $\tau_{a}$ is also considered in equation (19).
TABLE I: Calculated, simulation and experimental estimation of parameters for 2-DOF manipulator.

\begin{tabular}{|c|c|ccc|}
\hline Var. & Equivalent & Cal. & Sim. & Exp. \\
\hline \hline$m_{11}$ & $\frac{1}{3} m_{1} l_{1}^{2}+\frac{1}{3} m_{2} l_{2}^{2}+m_{2} l_{1}^{2}$ & 0.442 & 0.442 & 0.590 \\
$m_{11}^{\prime}$ & $m_{2} l_{1} l_{2}$ & 0.028 & 0.027 & 0.027 \\
$m_{12}$ & $\frac{1}{3} m_{2} l_{2}^{2}$ & 0.009 & 0.008 & 0.006 \\
$m_{12}^{\prime}$ & $\frac{1}{2} m_{2} l_{1} l_{2}$ & 0.014 & 0.015 & 0.010 \\
$n_{11}$ & $\frac{1}{2} m_{2} l_{1} l_{2}$ & 0.014 & 0.014 & 0.216 \\
$n_{12}$ & $m_{2} l_{1} l_{2}$ & 0.030 & 0.028 & 0.041 \\
$f_{11}$ & - & 0.001 & 0.004 & 0.001 \\
$m_{21}$ & $\frac{1}{3} m_{2} l_{2}^{2}$ & 0.009 & 0.009 & 0.005 \\
$m_{21}^{\prime}$ & $\frac{1}{2} m_{2} l_{1} l_{2}$ & 0.014 & 0.015 & 0.009 \\
$m_{22}$ & $\frac{1}{3} m_{2} l_{2}^{2}$ & 0.222 & 0.222 & 0.282 \\
$n_{21}$ & $\frac{1}{2} m_{2} l_{1} l_{2}$ & 0.014 & 0.013 & 0.282 \\
$f_{22}$ & - & 0.001 & 0.006 & 0.001 \\
\hline
\end{tabular}

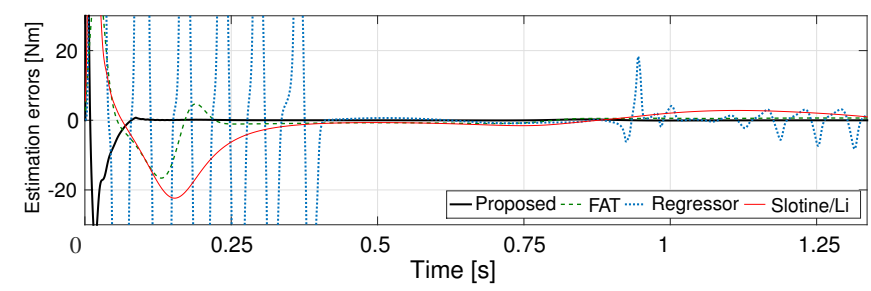

Fig. 8: Error $(\tilde{\phi})$ comparison of all the four controllers for the first joint angle of 2-DOF robot. As shown, the error for the FAT, regressor and Slotine/Li's controllers are not zero.

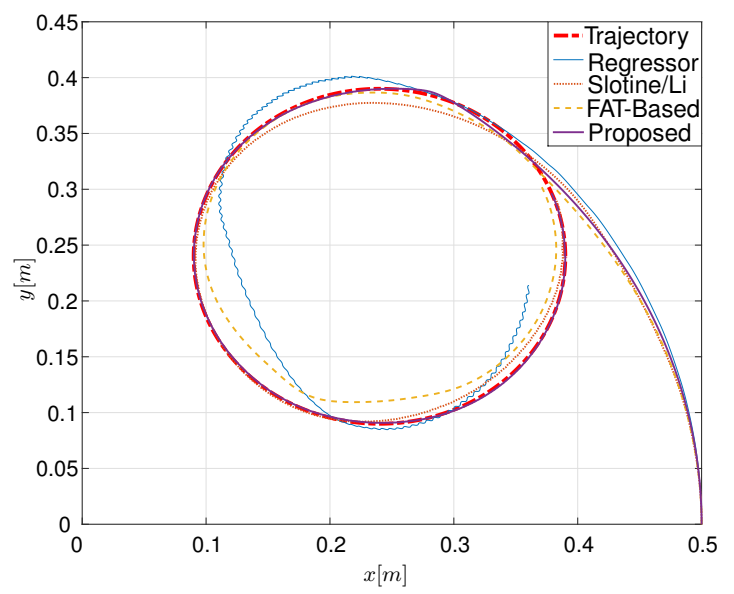

Fig. 9: Experimental results: position of end effectors starting from $0.5 \widehat{x}+0 \widehat{y} \mathrm{~m}$

\section{Model identification}

The Second part of the analysis consists of identifying the system parameters. Using the least square approximation (25) with an adequate excitation trajectory (24) along with the circular trajectory, the unknown parameters in the above matrices are estimated as shown in Table I. Both in simulations and experiments, $\tau_{a}$ is ignored for the identification process. The simulation results for parameter estimations produce very good results as shown in Table I. However, because in experiments, the real model is not known, we can only assume that the experimentally identified parameters must be 


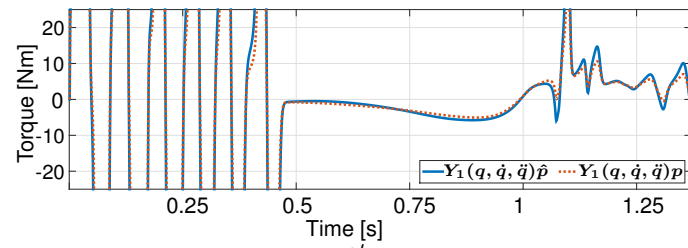

(a) First joint: $\boldsymbol{Y}_{\mathbf{1}}$ is the $1^{\text {st }}$ row of regressor matrix (8)

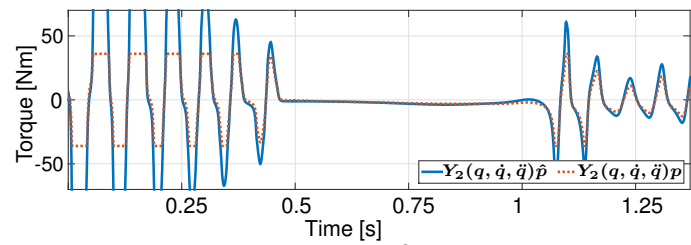

(c) Second Joint: $Y_{2}$ is the $2^{\text {nd }}$ row of regressor matrix

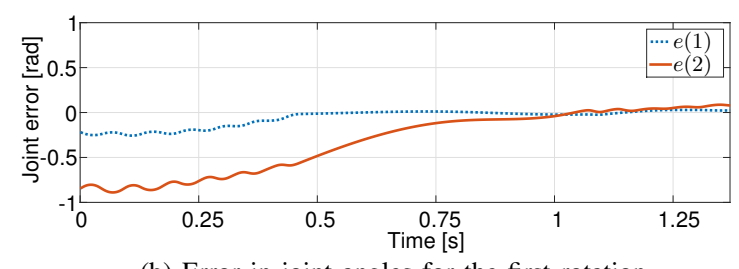

(b) Error in joint angles for the first rotation.

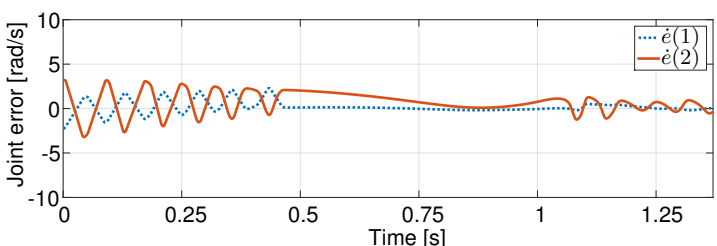

(d) Derivative of error in joint angles for the first rotation.

Fig. 4: Results of regressor-based adaptive control for $\omega=4 \mathrm{rad} / \mathrm{s}$. This technique requires inverse of inertia matrix $(\hat{M})$. Tracking of the desired trajectory is very sensitive to any change in control parameters.

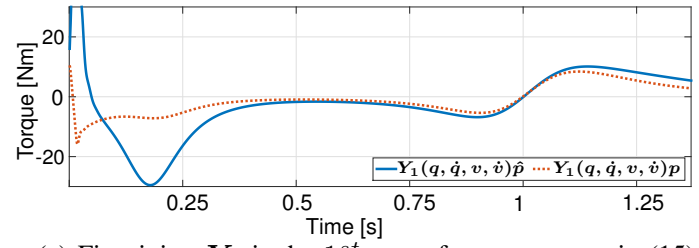

(a) First joint: $\boldsymbol{Y}_{\mathbf{1}}$ is the $1^{\text {st }}$ row of regressor matrix (15)

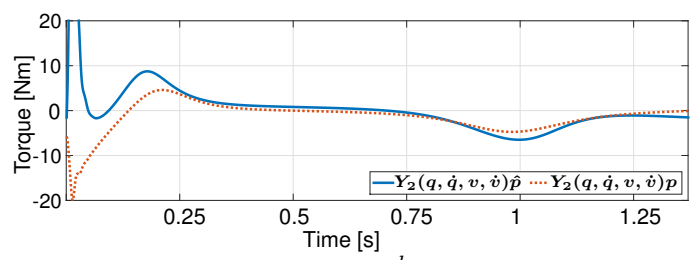

(c) Second joint: $\boldsymbol{Y}_{\mathbf{2}}$ is the $2^{\text {nd }}$ row of regressor matrix

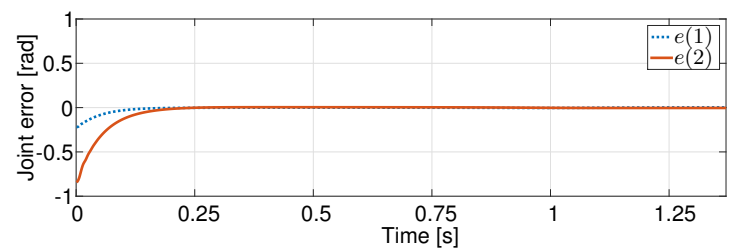

(b) Error in joint angles for the first rotation.

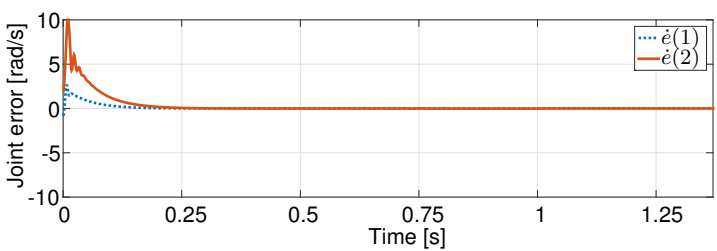

(d) Derivative of error in joint angles for the first rotation.

Fig. 5: Results of Slotine and Li's adaptive control for $\omega=4 \mathrm{rad} / \mathrm{s}$. As clear from Fig. (a) and (c), the estimated model dynamics do not follow the original model. Reason is that Lyapunov function only shows the tracking error to be a decreasing function.

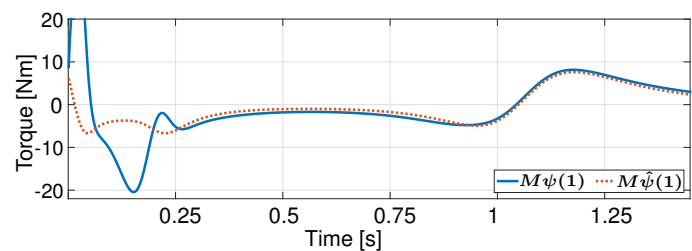

(a) First joint angle (18)

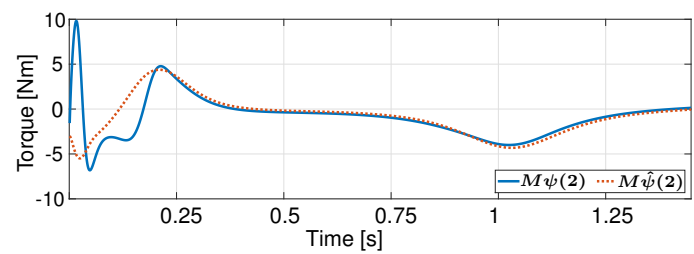

(c) Second joint angle

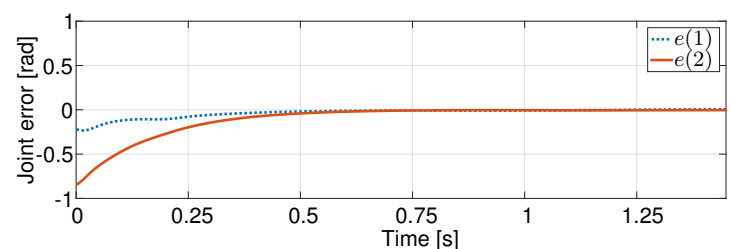

(b) Error in joint angles for the first rotation

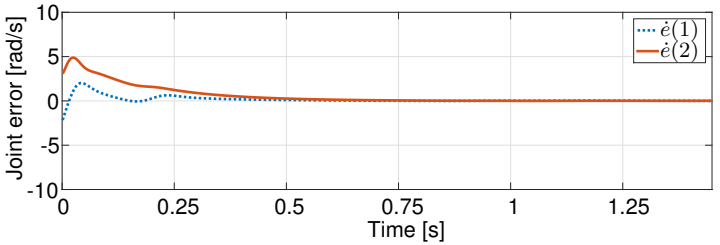

(d) Derivative of error in joint angles for the first rotation.

Fig. 6: Results of FAT-based adaptive control for $\omega=4 \mathrm{rad} / \mathrm{s}$. Approximation of system parameters by a linear combination of some orthogonal bases has high computational cost. 


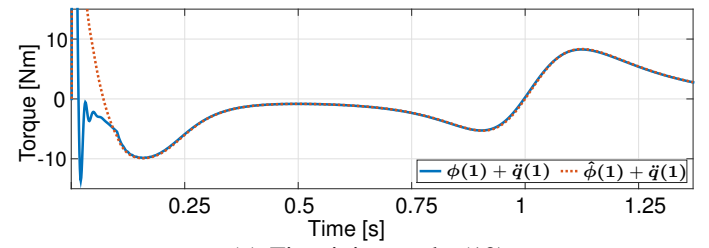

(a) First joint angle (19)

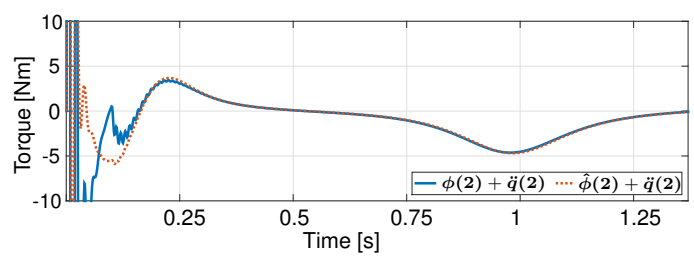

(c) Second joint angle

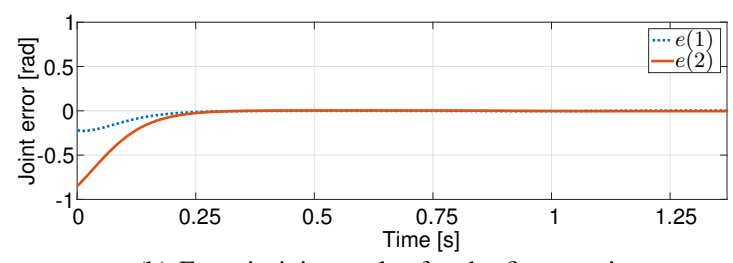

(b) Error in joint angles for the first rotation.

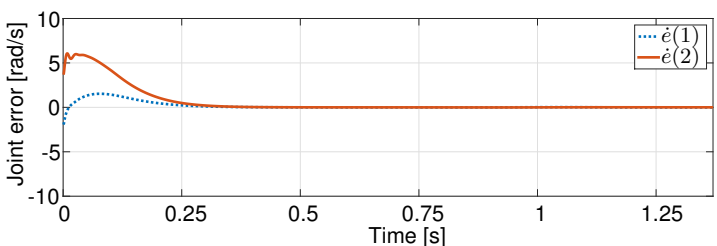

(d) Derivative of error in joint angles for the first rotation.

Fig. 7: Results of proposed adaptive control for $\omega=4 \mathrm{rad} / \mathrm{s}$. Taking a very low time constant for the update law will confirm the modeling error to approach zero. This technique requires only one parameter for the adaptation of system dynamics.

close to the calculated values. The identification process can be improved by adding torque boundedness and unmodeled parameters in terms of disturbances.

\section{CONCLUSIONS}

The proposed adaptive control technique guarantees convergence of any mismatch in the system by adjusting a single control parameter. Once the mismatch is eliminated, there is an accurate feedback linearization and a simple PD controller will achieve the desired performance. Using the adaptive control law, the model parameters are approximated applying least square approximation. After the identification of system parameters, the adaptive controller can be replaced by a simple PD controller. Compared to existing algorithms involving regressor-based approaches of FAT, the presented algorithm requires less computational cost. Furthermore, design prarmeters are less and more easily tuned in the proposed algorithm. Both simulation and experimental results show better and more precise tracking performance as compared to existing adaptive methods.

\section{REFERENCES}

[1] S. R. Munasinghe, M. Nakamura, S. Aoki, S. Goto, and N. Kyura, "High speed precise control of robot arms with assigned speed under torque constraint by trajectory generation in joint co-ordinates," IEEE International Conference on Systems, Man, and Cybernetics, vol. 2, pp. 854-859, 1999.

[2] V. Mhase, R. Sudarshan, O. Pardeshi, and P. V. Suryawanshi, "Integrated speed-position tracking with trajectory generation and synchronization for 2-axis dc motion control," International Journal of Engineering Research and Development, vol. 1, pp. 61-66, 2012.

[3] M. I. Ullah, S. A. Ajwad, R. U. Islam, U. Iqbal, and J. Iqbal, "Modeling and computed torque control of a 6 degree of freedom robotic arm," International Conference on Robotics and Emerging Allied Technologies in Engineering (iCREATE), pp. 133-138, 2014.

[4] J. J. Craig, Introduction to robotics: mechanics and control. Pearson Prentice Hall Upper Saddle River, 2005, vol. 3.

[5] C. C. De Wit, H. Olsson, K. J. Astrom, and P. Lischinsky, "A new model for control of systems with friction," IEEE Transactions on Automatic Control, vol. 40, no. 3, pp. 419-425, 1995.
[6] A. C. Bittencourt and S. Gunnarsson, "Static friction in a robot jointmodeling and identification of load and temperature effects," Journal of Dynamic Systems, Measurement, and Control, vol. 134, no. 5, 2012.

[7] S. H. Chong and K. Sato, "Practical and robust control for precision positioning systems," IEEE International Conference on Mechatronics (ICM), pp. 961-966, 2011.

[8] C. Kim and K. Lee, "Robust control of robot manipulators using dynamic compensators under parametric uncertainty," International Journal of Innovative Computing, Information and Control, vol. 7, no. 7, pp. 4129-4137, 2011.

[9] C. Y. Kai and A. C. Huang, "A regressor-free adaptive controller for robot manipulators without Slotine and Li's modification," Robotica, vol. 31, no. 07, pp. 1051-1058, 2013.

[10] M. C. Chien and A. C. Huang, "Adaptive impedance controller design for flexible-joint electrically-driven robots without computation of the regressor matrix," Robotica, vol. 30, no. 01, pp. 133-144, 2012.

[11] A. C. Huang, S. C. Wu, and W. F. Ting, "A FAT-based adaptive controller for robot manipulators without regressor matrix: theory and experiments," Robotica, vol. 24, no. 02, pp. 205-210, 2006.

[12] S. Jung and T. C. Hsia, "Neural network impedance force control of robot manipulator," IEEE Transactions on Industrial Electronics, vol. 45, no. 3, pp. 451-461, 1998.

[13] B. Achili, B. Daachi, Y. Amirat, A. Ali-Cherif, and M. E. Daachi, "A stable adaptive force/position controller for a C5 parallel robot: a neural network approach," Robotica, vol. 30, no. 07, pp. 1177-1187, 2012.

[14] R. Hedjar and P. Boucher, "Nonlinear receding-horizon control of rigid link robot manipulators," International Journal of Advanced Robotic Systems, vol. 2, no. 1, pp. 15-24, 2005.

[15] Z. J. Yang, H. Tsubakihara, S. Kanae, K. Wada, and C. Y. Su, "A novel robust nonlinear motion controller with disturbance observer," IEEE Transactions on Control Systems Technology, vol. 16, no. 1, pp. 137147, 2008.

[16] J. J. Slotine and W. Li, "On the adaptive control of robot manipulators," The international journal of Robotics Research, vol. 6, no. 3, pp. 49-59, 1987.

[17] C. D. Sousa and R. Cortesão, "Physical feasibility of robot base inertial parameter identification: A linear matrix inequality approach," The International Journal of Robotics Research, vol. 33, no. 6, pp. 931944, 2014.

[18] J. Swevers, C. Ganseman, D. B. Tükel, J. De Schutter, and H. Van Brussel, "Optimal robot excitation and identification," IEEE Transactions on Robotics and Automation, vol. 13, no. 5, pp. 730-740, 1997.

[19] B. Siciliano, L. Sciavicco, L. Villani, and G. Oriolo, Robotics: modelling, planning and control. Springer Science \& Business Media, 2009.

[20] W. Khalil and E. Dombre, Modeling, identification and control of robots. Butterworth-Heinemann, 2004. 